\title{
Recenser les personnages et conter les rapports de genre dans les manuels scolaires de mathématiques en Tunisie
}

Ibtihel Bouchoucha et Thérèse Locoh

\section{OpenEdition}

\section{Journals}

Édition électronique

URL : http://journals.openedition.org/trema/2668

DOI : $10.4000 /$ trema.2668

ISSN : 2107-0997

Éditeur

Faculté d'Éducation de l'université de Montpellier

Édition imprimée

Date de publication : 1 décembre 2011

Pagination : $128-141$

ISSN : 1167-315X

Référence électronique

Ibtihel Bouchoucha et Thérèse Locoh, «Recenser les personnages et conter les rapports de genre dans les manuels scolaires de mathématiques en Tunisie », Tréma [En ligne], 35 - 36 | 2011, mis en ligne le 01 décembre 2013, consulté le 01 mai 2019. URL : http://journals.openedition.org/trema/2668 ; DOI : 10.4000/trema.2668

Ce document a été généré automatiquement le 1 mai 2019.

Trema 
Recenser les personnages et conter les rapports de genre dans les manuels scolaires de mathématiques en Tunisie

Ibtihel Bouchoucha et Thérèse Locoh

I. Introduction 
1 Durant le demi-siècle qui vient de s'écouler, sous l'effet de l'impulsion politique donnée par le code de statut personnel (1956), puis grâce aux progrès rapides du pays, notamment en matière d'instruction féminine, la Tunisie a fait des pas importants vers l'égalité entre hommes et femmes. Mais les esprits et les mentalités sont encore imprégnés d'une perception traditionnelle tributaire de l'héritage social et culturel de la Tunisie, transmis d'une manière directe et indirecte aux jeunes générations (BOUCHOUCHA et LOCOH, 2008). Ces conceptions sont, d'une manière directe et indirecte, transmises aux jeunes générations encore très influencées par une conception traditionnelle des rôles et statuts sexués malgré les changements dans l'éducation

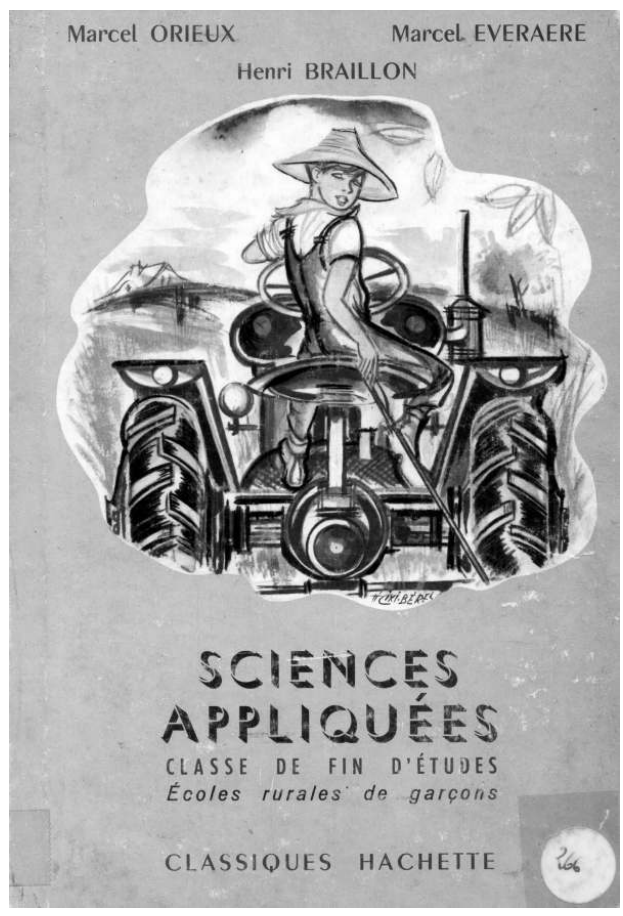
et le quotidien de la société tunisienne.

2 Les manuels scolaires contribuent puissamment à la transmission des normes et des valeurs. Ils jouent ainsi un rôle primordial dans la construction de soi, le développement de la personnalité par les modèles transmis implicitement ou explicitement. Plusieurs recherches ont étudié cette relation enfant/manuel et ont insisté sur l'importance du rôle joué par cet instrument didactique dans l'élaboration des normes et des valeurs chez les enfants, et notamment les publications du programme de recherche initié par Carole BRUGEILLES et Sylvie CROMER sur les manuels africains (BOUCHOUCHA et LOCOH, 2008 ; CROMER et $\mathrm{LOCOH}, 2008$ ). Les différentes études menées ont illustré la persistance, dans les manuels proposés aux élèves, de représentations inégalitaires des sexes.

3 C'est ce que nous observons dans ce travail, à partir de l'analyse des représentations des personnages dans les textes d'une série de livres de mathématiques correspondant aux niveaux scolaires du premier cycle de l'enseignement de base (primaire) tunisien. Ces manuels, identiques pour tous les élèves des écoles publiques et privées, constituent la seule collection officielle utilisée par les élèves et par les enseignants durant l'année scolaire 2003-2004. Les manuels de la première année jusqu'à la quatrième année intitulés Livre de mathématique constituent une nouvelle collection élaborée dans le cadre du programme « compétences de base ». Les manuels de la cinquième année et de la sixième année intitulés Mathématique de base font partie d'une collection révisée, élaborée dans le cadre du programme « l'école de base » en 2000-2001.

Par l'analyse quantitative et qualitative des différentes variables qui définissent ces personnages (sexe, âge, activités, relations,...), nous montrons comment les hommes et les femmes, les filles et les garçons sont identifiés dans les manuels scolaires et comment la question de l'égalité entre les sexes y est implicitement abordée. En observant comme une " population » particulière l'ensemble des personnages individuels présents dans les textes des manuels scolaires, nous voulons répondre aux trois questions suivantes: comment évoluent, au fil du cursus du primaire, les représentations des rôles sexués dans les manuels ? Prennent-elles en compte les changements de la société tunisienne ou, au 
contraire, ont-elles tendance à continuer de valoriser des modèles traditionnels qui excluent les femmes de la sphère publique et les cantonnent dans leur rôle reproductif ? Comment sont présentées les relations entre les deux sexes, enfants et adultes, dans les textes des manuels scolaires?

La méthodologie utilisée, celle développée par Carole BRUGEILLES et Sylvie CROMER (2005), consiste à recenser de manière exhaustive, à l'aide d'une grille, tous les personnages d'un manuel scolaire ainsi que les caractéristiques de son sexe social, à savoir concernant notre étude le sexe, l'âge, mais aussi les désignations, les métiers et les relations.

\section{Dénombrement des personnages et déséquilibre des sexes}

6 Pour les élèves de l'enseignement primaire tunisien, le livre scolaire constitue aujourd'hui le premier matériel didactique malgré le développement d'autres instruments audio-visuels. Il est souvent utilisé de manière répétitive durant toute l'année (cours, exercices). Il est composé d'un ensemble de textes et d'images, dans lesquels apparaissent des personnages, tantôt collectifs (groupes de personnages) tantôt individuels. Dans ce travail, nous focalisons notre analyse sur les personnages individuels dans les textes.

7 Dans ces derniers, nous avons recensé 1248 personnages individuels de sexe et d'âge déterminés. Si les générations s'équilibrent, le rapport des sexes est fortement déséquilibré. On compte 572 enfants et 676 adultes, soit une légère majorité d'adultes qui représente $54 \%$ des personnages. Mais on dénombre 308 personnages féminins pour 940 personnages masculins, soit $75 \%$ de personnages masculins: les trois quarts des personnages individuels sont des hommes ou des garçons et le sexe féminin ne compte que pour un quart d'humanité fictive !

8 La dominance masculine et, parallèlement, l'occultation féminine s'accroissent fortement avec l'âge et le niveau scolaire. Les personnages féminins représentent $34 \%$ des enfants (195 filles et 377 garçons) et seulement 17\% des adultes ( 113 femmes et 557 hommes). Les hommes sont cinq fois plus nombreux que les femmes et les garçons deux fois plus nombreux que les filles. Cette suprématie numérique masculine, notamment au niveau des adultes, s'affirme de la première à la dernière année du primaire (figure 1). À partir de la 3e année, la proportion des hommes est supérieure à celle des garçons. En revanche, du côté féminin, les deux populations, filles et femmes, diminuent et les écarts entre elles se réduisent à partir de la 3e année. Les femmes, quel que soit le niveau scolaire, constituent toujours moins de $10 \%$ des personnages. 
Figure 1. Répartition des personnages selon le sexe, l'âge et le niveau scolaire (en \%)

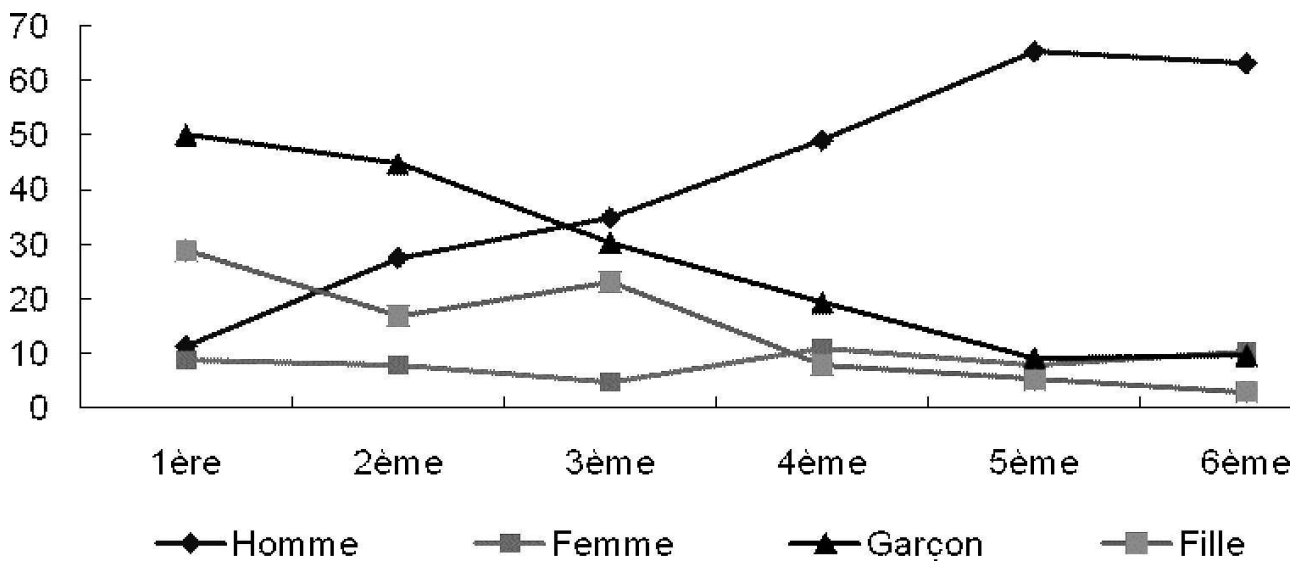

Source : Données collectées par les auteures

9 Ces premiers résultats d'un recensement purement numérique témoignent bien que les manuels scolaires véhiculent une représentation d'un ordre social qui ne correspond nullement à la réalité tunisienne. Clairement cette forte et donc perceptible inégalité numérique entre les deux sexes, surtout parmi les adultes, est un signe fort pour les élèves, compte tenu du rôle de modèle que jouent les adultes pour les enfants, dans la vie comme à travers les manuels : le sexe dominant est le sexe masculin. En avançant dans le cursus scolaire, plus les concepteurs puisent leurs exemples dans la vie active, sans doute pour préparer les enfants au monde des adultes, plus la présence féminine diminue et celle des hommes s'accroît. Ces déséquilibres influencent de manière directe ou indirecte les perceptions que se font les jeunes de leur place future dans la société et du rôle qu'ils auront à y jouer.

De surcroît, la disparité entre les sexes n'est pas seulement quantitative, elle est aussi qualitative : elle concerne les situations dans lesquelles les personnages sont représentés.

\section{Les personnages adultes : désigner pour assigner}

11 Les modes de désignation des personnages sont très variés et confèrent aux personnages une place dans la société fictive des manuels. Un personnage peut être identifié par un prénom (Mohamed, Leila...), un patronyme ou une civilité (Monsieur, Madame...), un lien de parenté (père, mère....), un autre lien (ami, collègue, voisine...), enfin un «statut ». L'identification par un statut peut revêtir plusieurs modalités: une fonction politique (ministre), une fonction sociale (élève), une profession (vendeur, institutrice) ou une possession (propriétaire). Malgré cette diversité, l'identification s'avère spécifique pour chaque sexe. Tout au long des manuels de l'enseignement primaire, les trois quarts des hommes sont désignés par un statut social, notamment une activité professionnelle (le maître, le médecin, un menuisier...). Ces désignations témoignent de leur intégration dans la vie active, même quand ils ont un faible niveau scolaire. En revanche, les trois quarts des femmes sont nommées par un lien de parenté, qui les renvoie à la sphère privée.

Ces désignations suggèrent aux élèves, filles comme garçons, une assignation sociale en fonction du sexe. D'où découle l'intérêt d'analyser plus précisément les activités professionnelles des personnages adultes. 


\section{Images de la vie active : la réalité dépasse la fiction} situation des femmes, depuis l'Indépendance. Le taux d'activité des femmes a augmenté, l'âge au premier mariage est beaucoup plus tardif, le taux d'analphabétisme de femmes a baissé, et, exemple frappant, actuellement les femmes sont plus nombreuses dans l'enseignement supérieur que les hommes. Les femmes ont commencé à investir le monde extérieur et elles sont aux côtés des hommes dans presque tous les domaines. Cette situation va, à n'en pas douter, influencer de façon toute nouvelle les aspirations éducatives et professionnelles des filles d'une part, des garçons d'autre part. Pourtant, cette nouvelle donne de la société tunisienne n'apparaît pas dans les manuels scolaires où les personnages féminins sont quasi invisibles, même dans les professions où actuellement elles ont une présence remarquable.

Figure 2. Répartition des professions évoquées selon le sexe du personnage (en \%)



Comme le montre le tableau 1, la différence entre le réel observé statistiquement et les représentations proposées aux élèves dans les manuels scolaires est notable. Celles-ci ne reflètent pas la réalité tunisienne, à savoir la présence féminine accrue, qualifiée et 
diversifiée dans l'activité économique tunisienne d'aujourd'hui. On peut s'étonner, par exemple, que soit minimisée dans des manuels scolaires la présence des institutrices (50 \% dans la société tunisienne de 2004, mais $21 \%$ seulement dans les manuels). Les femmes médecins, actuellement $37 \%$ du corps médical, sont aussi absentes. Les agricultrices et vendeuses sont très rares, respectivement $2 \%$ et $1 \%$, alors que dans le recensement de 2004 on en comptait respectivement $31,7 \%$ et $11,3 \%$ dans ces professions.

Tableau 1. Proportion de femmes dans différentes professions, selon le recensement (2004) et dans le monde des manuels (en \%)

\begin{tabular}{|l|c|l|}
\hline & \multicolumn{1}{|c|}{$\begin{array}{l}\text { La réalité de la } \\
\text { société tunisienne (\%) }\end{array}$} & $\begin{array}{l}\text { Les représentations des } \\
\text { manuels (\%) }\end{array}$ \\
\hline Proportion d'agricultrices & $31,7^{*}$ & 1,9 \\
\hline $\begin{array}{l}\text { Proportion d'enseignantes } \\
\text { dans l'enseignement } \\
\text { primaire }\end{array}$ & 50,8 & 20,8 \\
\hline $\begin{array}{l}\text { Proportion de femmes } \\
\text { médecins }\end{array}$ & 37,0 & 0,0 \\
\hline Proportion de vendeuses & 11,3 & 0,9 \\
\hline $\begin{array}{l}\text { *Lecture du tableau : sur 100 agriculteurs dans la population tunisienne, } 31,7 \% \text { sont des femmes, alors que } \\
\text { dans les représentations des manuels scolaires étudiés sur 100 agriculteurs } 1,9 \% \text { sont des femmes. }\end{array}$ \\
\hline
\end{tabular}

Colonne 1 : La réalité de la société tunisienne (\%)

Colonne 2 : Les représentations des manuels (\%) ${ }^{2}$

16 Cette occultation de la présence féminine dans la vie économique est manifeste également dans les relations que nous allons exposer.

\section{V. Être seul ou en relation, reflet d'un monde doublement ségrégué}

Dans le corpus étudié, on recense 1605 relations entre les 1248 personnages individuels : 1460 relations impliquent au moins un personnage masculin; 656 relations seulement impliquent au moins un personnage féminin. Le nombre très élevé de relations avec au moins un individu masculin s'explique par la dominance numérique masculine observée. Globalement, les personnages, masculins ou féminins, enfant ou adulte, sont plus souvent en relation que seuls ( $68 \%$ sont en relation), avec des différences notables selon l'âge et le sexe (tableau 2). Enfants et personnages féminins, numériquement moins importants, sont plus souvent inclus dans des relations. Si la différence entre filles et garçons n'est que de 4 points, l'écart entre hommes et femmes est remarquable : il est de 14 points. De fait, les hommes des manuels scolaires apparaissent dans les deux types de configurations, en interrelation avec les autres et presque autant, acteurs indépendants. Seules 3 femmes sur 10 sont montrées seules. Etre présenté seul peut être interprété comme une marque d'autonomie du personnage. L'examen des relations peut mettre en évidence la place et l'importance accordées au personnage dans la société fictive et le degré de mixité - ou de ségrégation - de cette dernière. 
Tableau 2. Proportion des personnages seuls et en relation selon le sexe et l'âge (en \%)

\begin{tabular}{|l|c|c|}
\hline \multicolumn{1}{|c|}{ Personnages } & En relation & Seul \\
\hline Filles & $81 \%$ & $19 \%$ \\
\hline Garçons & $77 \%$ & $23 \%$ \\
\hline Femmes & $71 \%$ & $29 \%$ \\
\hline Hommes & $57 \%$ & $43 \%$ \\
\hline
\end{tabular}

Source : Données collectées par les auteures

Dans l'analyse qui suit, ne sont considérées que les relations entre personnages dont le sexe et l'âge sont connus ${ }^{3}$, soit 1366 relations. Il s'agit de savoir quels types et natures de relations les uns et les autres ont la possibilité de développer: sont-ce des relations intergénérationnelles ou entre pairs? Mixtes ou non? Que font les personnages ensemble?

Le tableau 3 répartit tous les types de relations possibles entre les personnages selon leur sexe et leur âge et permet de mettre en évidence le degré de cohabitation des âges et des sexes.

Tableau 3 : Relations entre les personnages selon leur sexe et leur âge en effectif (en \%)

\begin{tabular}{|c|c|c|c|}
\hline \multicolumn{4}{|l|}{ RELATIONS ENTRE ENFANTS } \\
\hline Filles-Filles & 83 & $6 \%$ & \\
\hline Garçons-Filles & 301 & $22 \%$ & \\
\hline Garçons-Garçons & 384 & $28 \%$ & \\
\hline Sous-total & 768 & & $56 \%$ \\
\hline \multicolumn{4}{|l|}{ RELATIONS ENTRE ADULTES } \\
\hline Femmes-Femmes & 8 & $1 \%$ & \\
\hline Hommes-Femmes & 67 & $5 \%$ & \\
\hline Hommes-Hommes & 263 & $19 \%$ & \\
\hline Sous-total & 338 & & $25 \%$ \\
\hline \multicolumn{4}{|c|}{ RELATIONS INTERGENERATIONNELLES } \\
\hline Fille-Femmes & 39 & $3 \%$ & \\
\hline Filles-Hommes & 53 & $4 \%$ & \\
\hline Garçons-Femmes & 41 & $3 \%$ & \\
\hline Garçons-Hommes & 127 & $9 \%$ & \\
\hline Sous-total & 260 & & $19 \%$ \\
\hline TOTAL & 1366 & $100 \%$ & \\
\hline
\end{tabular}

Source : Données collectêes par les auteures

\section{1 Des relations entre pairs d'âge favorisées}

En considérant le tableau 3, il apparait que $81 \%$ des relations se déroulent entre personnages de même génération, et plus particulièrement entre enfants. $56 \%$ des relations sont en effet des relations entre enfants, soit plus d'une relation sur deux concerne deux enfants. C'est une première ségrégation qui existe dans la société des manuels : entre le monde de l'enfance et le monde des adultes. Est-ce l'école qui sépare 
les générations? Pourtant les manuels de mathématiques tunisiens ne montrent pas exclusivement les enfants en situation scolaire comme nous le verrons par la suite. Les relations intergénérationnelles ne représentent que 19\% des relations et concernent essentiellement les garçons et les hommes, les autres types relations étant marginales et représentant moins de $10 \%$ des relations.

En fait, trois relations se détachent nettement: les relations entre garçons ( $28 \%$ des relations), puis entre garçons et filles (22\% relations), enfin entre hommes (19\% relations). De fait, les relations bénéficient essentiellement aux garçons : 6 relations sur 10 impliquent un garçon (tableau 3, 62\% des relations), alors que les hommes et les filles sont concernés respectivement par $37 \%$ et $35 \%$ des relations. Les femmes sont les grandes déficitaires: elles apparaissent dans une relation sur 10. À quoi servent les relations sociales ? Elles permettent de se construire dans son rapport à l'Autre, que cet Autre soit un pair, une autre génération, l'autre sexe. Ainsi les manuels privilégient la mise en scène des relations enfantines qui permettent la construction de soi et la projection vers l'âge adulte. Mais en même temps ils légitiment le fait que c'est aux personnages garçons que doivent profiter toutes ces expériences, avec leurs pairs et avec des hommes : ne serontils pas les acteurs dominants de la société de demain, puisque les personnages hommes sont numériquement plus nombreux? Mais pour les filles, ce travail de socialisation ne semble pas nécessaire... D'ailleurs les femmes, auxquelles elles sont censées s'identifier n'apparaissent pas comme des interlocutrices utiles, sinon avec des hommes: le rôle de mère n'est donc pas particulièrement valorisé.

\section{2 Des relations masculines homosexuées versus des relations mixtes pour les personnages féminins}

21 La mixité des manuels est trompeuse, comme le montre le tableau 4, qui calcule le degré de mixité sexuelle des relations pour chacun des personnages en fonction de son sexe et de son âge : la mixité est préconisée pour les personnages féminins, mais accessoire pour les masculins. De quelle mixité s'agit-il donc?

Les personnages féminins sont présentés majoritairement dans des relations mixtes : 74\% des relations avec des filles et $70 \%$ des relations avec des femmes sont mixtes. De fait l'entre soi féminin est minoritaire et il est particulièrement inexistant entre les femmes (8 relations entre femmes seulement tout au long du cursus scolaire, soit $1 \%$ des relations, tableau 3). Ainsi les personnages féminins sont doublement dépendantes : filles et femmes sont à la fois moins montrées "seules" comme actrices à part entière, et elles n'interagissent guère entre elles : elles ne sont envisagées qu'en étant « en relation » avec des masculins : à disposition? Sous contrôle?

Les personnages masculins en revanche - plus nombreux - sont doublement autonomes et cette indépendance augmente avec l'âge : à la fois plus souvent seuls, comme nous l'avons vu, et plus souvent inclus dans des relations homosexuées (60\% pour les garçons et $76 \%$ pour les hommes) que dans des relations mixtes. Si les garçons bénéficient malgré tout de relations mixtes ( $40 \%$ des relations incluant un garçon sont mixtes), seulement 24 $\%$ des relations impliquent des hommes dans des relations mixtes. C'est la seconde ségrégation: celle entre les sexes. Ou plus exactement les personnages masculins bénéficient d'un espace masculin réservé, alors que les personnages féminins n'existent qu'en relation avec des personnages masculins. Il n'y a pas de réelle mixité égalitaire, entendue au sens de partage d'espaces communs par les personnages. On peut se 
demander si la mixité imposée aux personnages féminins n'est pas synonyme de dépendance aux hommes.

Tableau 4 : Relations des personnages, en fonction du sexe et de l'âge des personnages (en effectif et en \%)

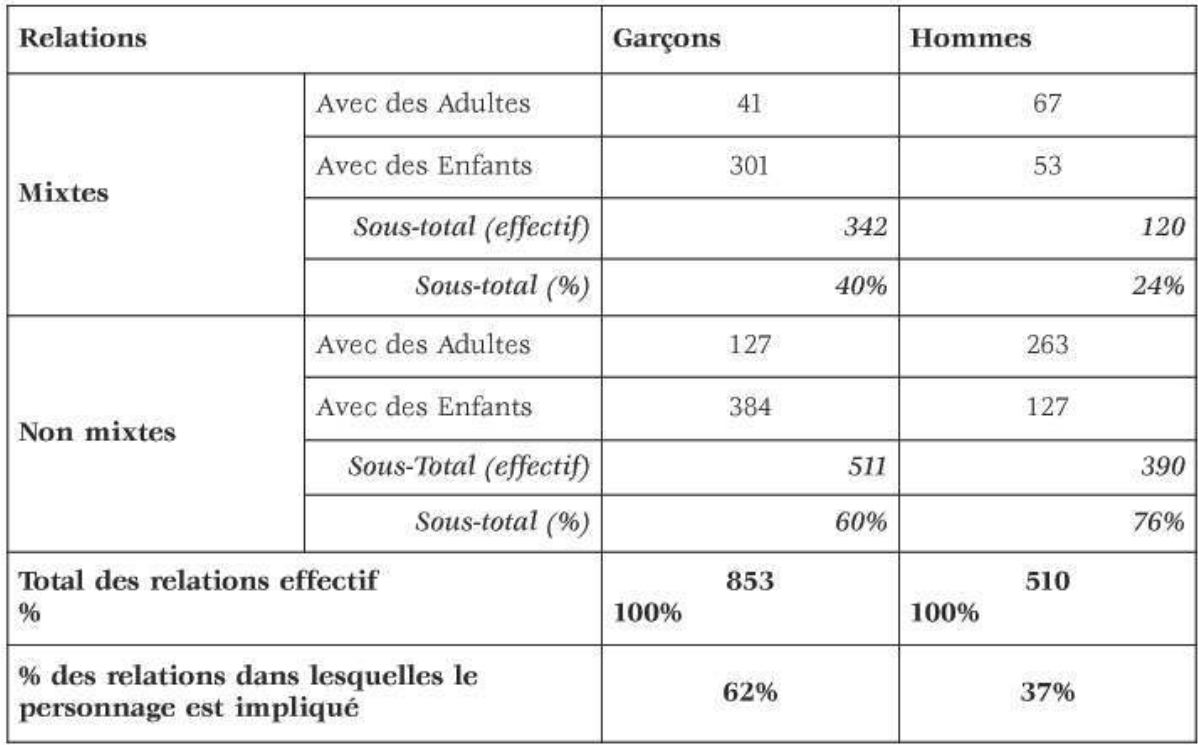

Source : Données collectées par les auteures

\section{3 Des interactions genrées}

Les personnages en relation peuvent être dans trois situations-types : en coprésence (sans interagir l'un avec l'autre), en interaction (par exemple deux enfants discutent ensemble) ou en comparaison (de la taille, du poids, de l'âge et/ou des objets possédés) introduisant l'idée d'évaluation et de compétition. Nous choisissons de jeter un éclairage particulier dans cette partie sur les relations d'interactions et leur nature. Ce choix est justifie par le fait que globalement nos personnages sont plus souvent en interaction (46\%) qu'en comparaison (38\%) ou en coprésence (19\%). Les interactions deviennent plus nombreuses dans les niveaux scolaires plus élevés.

Les personnages féminins sont plus souvent placés dans des relations d'interaction (41\% des filles et $59 \%$ des femmes sont en interaction contre $39 \%$ des garçons et $56 \%$ des hommes). Cinq interactions majeures ont été recensées : la sociabilité (discuter, échanger, rendre visite), l'interaction scolaire entre enfants, l'interaction professionnelle qui peut inclure des enfants, le jeu, le fait de mener ensemble une tâche (un achat, une tâche domestique...). Il est notable, qu'à l'exception du jeu, dont sont exclues les femmes et l'interaction professionnelle dont sont exclues les filles, les autres interactions ne sont pas l'apanage d'un sexe en particulier. Pour autant, comme le montre le tableau 5, les interactions sont genrées, c'est-à-dire que les personnages ne sont pas impliqués, selon leur sexe, avec la même intensité dans les interactions et cela confirme les précédents résultats. 
Tableau 5 : Nature des relations selon le sexe et l'âge du personnage (en effectif et en \%)

\begin{tabular}{|l|c|c|c|c|c|c|c|c|}
\hline & \multicolumn{2}{|c|}{ Garçons } & \multicolumn{2}{c|}{ Filles } & \multicolumn{2}{c|}{ Hommes } & \multicolumn{2}{c|}{ Femmes } \\
\hline & Effectif & $\%$ & Effectif & $\%$ & Effectif & $\%$ & Effectif & $\%$ \\
\hline $\begin{array}{l}\text { Sociabilité } \\
\text { (visiter, partager...) }\end{array}$ & 83 & $26 \%$ & 77 & $42 \%$ & 79 & $28 \%$ & 42 & $48 \%$ \\
\hline $\begin{array}{l}\text { Interaction } \\
\text { scolaire }\end{array}$ & 39 & $12 \%$ & 26 & $14 \%$ & - & - & - & - \\
\hline $\begin{array}{l}\text { Interaction } \\
\text { ludique }\end{array}$ & 64 & $20 \%$ & 28 & $15 \%$ & 12 & $4 \%$ & 0 & $0 \%$ \\
\hline $\begin{array}{l}\text { Mener à bien une } \\
\text { tâche ensemble }\end{array}$ & 100 & $31 \%$ & 52 & $28 \%$ & 26 & $9 \%$ & 16 & $18 \%$ \\
\hline $\begin{array}{l}\text { Interaction } \\
\text { professionnelle }\end{array}$ & 36 & $11 \%$ & 2 & $1 \%$ & 159 & $57 \%$ & 29 & $33 \%$ \\
\hline Total & 322 & $100 \%$ & 185 & $100 \%$ & 278 & $100 \%$ & 87 & $100 \%$ \\
\hline
\end{tabular}

Source : Données collectées par les auteures traditionnel de "féminité ", c'est-à-dire une caractéristique imputée au sexe féminin. L'activité professionnelle vient en tête chez les hommes. Les garçons ont le privilège de bénéficier de manière équilibrée des interactions majeures et notamment de «faire avec ».

Il est maintenant intéressant de vérifier la nature de l'interaction en fonction des personnages impliqués. Sont prises en considération les interactions les plus nombreuses et les plus significatives. enfants. La mixité conduit les filles et les garçons à partager des tâches et à exercer leur sociabilité, mais ensemble filles et garçons sont moins dans le jeu. C'est l'entre soi masculin, entre pairs, qui permet aux garçons de développer leurs compétences ludiques. Par leurs relations avec les hommes (figure 3b), les garçons sont inclus dans des activités professionnelles (aider aux travaux des champs par exemple).

Figure 3a. Répartition des interactions selon la nature de l'interaction et le sexe des enfants (en \%)

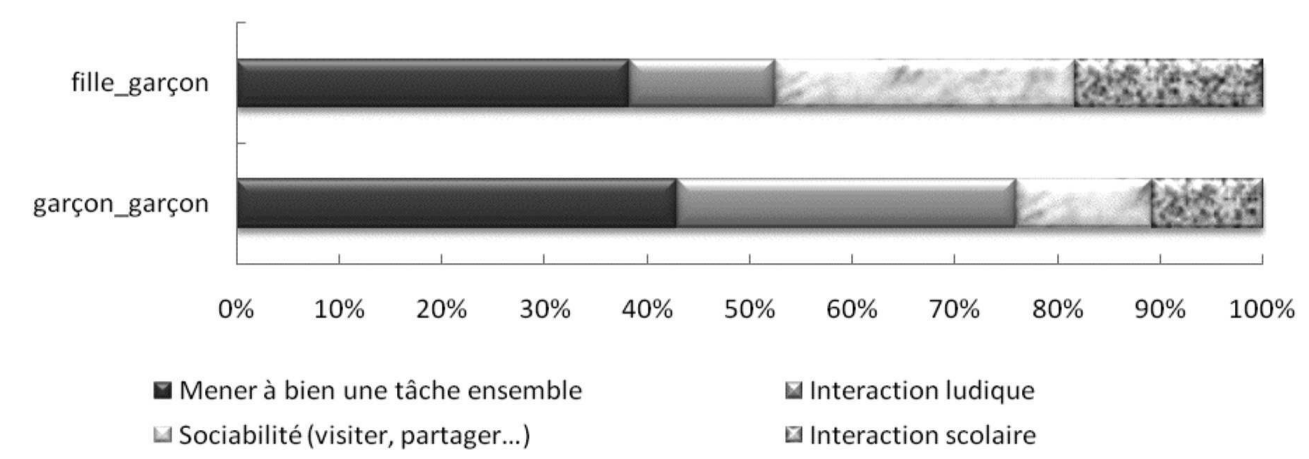

Source : Données collectées par les auteures

Les hommes entre eux exercent majoritairement une activité professionnelle ou de la sociabilité. Ceci peut conduire les élèves à penser que l'activité économique est une affaire d'hommes. D'autant que les femmes ne sont incluses dans des relations 
professionnelles qu'avec des hommes: il n'existe pas d'interactions professionnelles entre femmes ni de femmes professionnelles seules.

Figure 3b. Répartition des interactions homosexuées masculines selon la nature de l'interaction et l'âge des personnages (en \%)

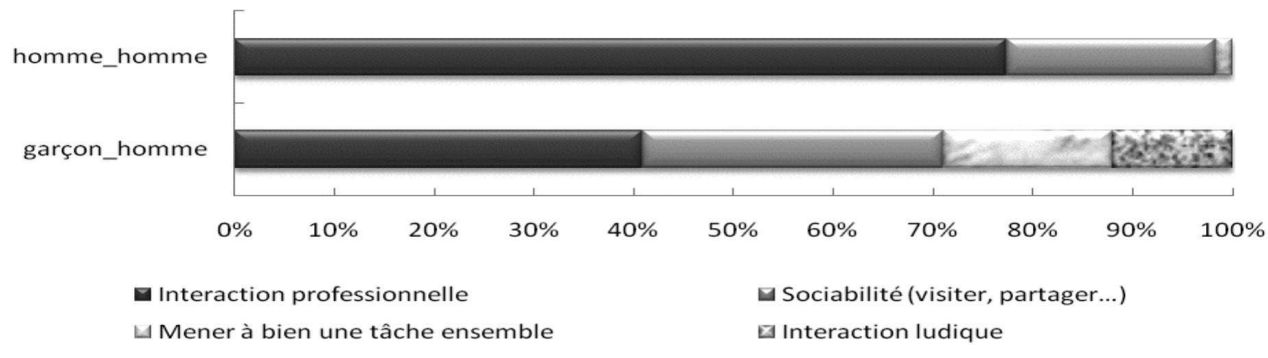

Source : Données collectées par les auteures

\section{Conclusion}

Grâce à une volonté affichée depuis 1956 par les autorités politiques, grâce aussi à la prise de conscience des Tunisiens et plus encore des Tunisiennes, et à leurs efforts, la Tunisie a fait des pas importants vers l'égalité entre hommes et femmes. Mais il reste beaucoup à faire dans la sensibilisation des esprits et des mentalités, encore soumis à une perception traditionnelle, tributaire de l'héritage social et culturel qui confine les femmes dans leur rôle d'épouse et de mère. L'analyse des manuels scolaires montre à l'évidence que le contenu des textes, pourtant inspiré de la vie quotidienne, ne prend pas en compte les changements qui se produisent dans la société et dans les rapports entre hommes et femmes. Les garçons et les hommes s'imposent comme les acteurs dominants. Cette suprématie s'exprime par l'imposante dominance numérique, par la multiplicité des relations possibles pour le garçon, par la place professionnelle majeure des hommes, par le privilège de l'entre soi masculin. Femmes et filles sont considérées comme une minorité, presque toujours en retrait ou n'ayant droit de cité sur la scène des manuels que par la médiation des hommes. Ce modèle que véhiculent les manuels scolaires limite le champ des avenirs possibles pour tous les enfants, garçons et filles. Il les enferme encore dans une perception traditionnelle de ce qu'ils deviendront. Les manuels recourent trop peu à des images diversifiées d'avenirs possibles pour stimuler leur imaginaire, surtout à cet âge où les enfants se rapprochent du monde des adultes et se préparent à entrer dans la vie active. Beaucoup reste à faire pour que les manuels cessent de perpétuer des images stéréotypées et « datées » des relations entre les sexes. 


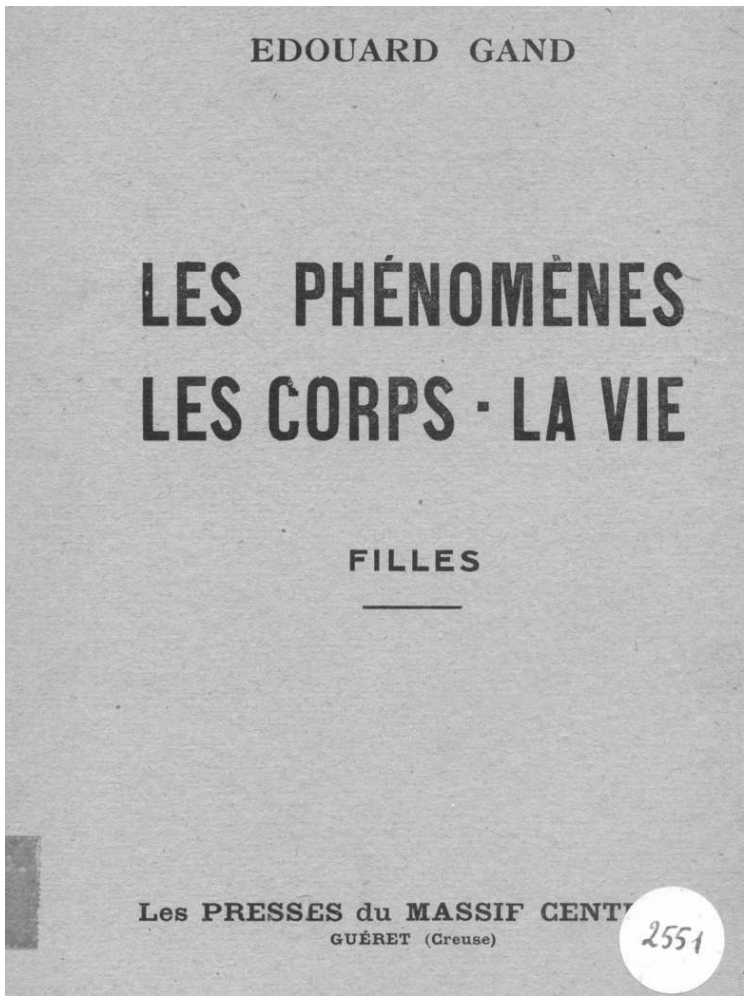

Les phénomènes - Les corps - La vie, Filles, Edouard GAND, Paris : Les presses du Massif Central, 360 p., Couverture - Source : CEDRHE.

\section{BIBLIOGRAPHIE}

BOUCHOUCHA I. et LOCOH T., Les manuels scolaires en Tunisie reflètent-ils la réalité tunisienne? Paris, CEPED, 2008

BRUGEILLES C., CROMER S. et Locoh T., Analyser les représentations sexuées dans les manuels scolaires. Paris, CEPED, 2008

BRUGEILLES C., CROMER S. Analyser les représentations du masculin et féminin dans les manuels scolaires. Paris, CEPED [Collection les Clefs Pour], 2005

Commission nationale Tunisienne pour L'éducation, la science et la culture secteur de l'éducation développement de l'éducation en Tunisie 1994-1996. UNESCO-ALESCO-ISESCO.

CREDIF, Centre de recherches, d'études, de documentation et d'information sur la femme. L'éducation selon le genre en Tunisie. Bulletin de l'Observatoire de la condition de la femme, 2001

CREDIF, Centre de recherches, d'études, de documentation et d'information sur la femme Le potentiel économique des femmes. Le contexte et les perspectives du travail féminin en Tunisie, 2000

Institut national de statistique, Les premiers résultats du recensement général de la population 2004, 2005 
MICHEL A., Non aux stéréotypes! Vaincre le sexisme dans les livres pour enfants et les manuels scolaires. UNESCO, 1986

Ministère de l'éducation nationale, Les fondamentaux de l'éducation et de l'enseignement scolaire. Tunis (en arabe), 2002

Ministère de l'éducation nationale. Vers le l'instauration de la société du savoir, la nouvelle réforme du système éducatif tunisien, Programme de mise en œuvre du projet "École de demain », 2002-2007, 2002

UNESCO, L'évaluation de l'éducation pour tous à l'an 2000 : rapport des pays, Tunisie, 2000

\section{Corpus des manuels scolaires étudiés}

BEHAJ E. et al., La Mathématique de base, pour les élèves de la 6e année de l'enseignement de base, Centre national pédagogique, 2003

ELAICH et al., La Mathématique de base, pour les élèves de la 5e année de l'enseignement de base, Tunis, Centre national pédagogique, 2003

ESSENEKLI et al., Mathématique, la 1re année de l'enseignement de base. Tunis, Centre national pédagogique, 2003

ESSAYAH et al., Mathématique, la 2e année de l'enseignement de base. Tunis, Centre national pédagogique, 2003

TAMER et al., Mathématique, la 3e année de l'enseignement de base. Tunis, Centre national pédagogique, 2003

TAMER et al., Mathématique, la 4e année de l'enseignement de base. Tunis, Centre national pédagogique, 2003

\section{NOTES}

1. Source : Recensement 2004

2. Source : Données collectées par les auteures

3. 239 relations concernent des personnages dont l'âge est indéfini.

\section{RÉSUMÉS}

Dès 1956, avec l'adoption du code de statut personnel, la Tunisie a tracé le chemin vers l'égalité entre hommes et femmes. Cependant, les esprits et les mentalités restent encore imprégnés d'une perception traditionnelle, tributaire de leur héritage socioculturel. Ces conceptions sont, d'une manière directe et indirecte, encore présentes dans de nombreuses productions culturelles, et notamment les manuels scolaires. C'est ce que montre l'analyse des représentations sexuées portées par les personnages présentés dans une série de manuels scolaires de mathématiques de l'enseignement primaire (2003-2004). 
Since the independence (1956), Tunisia has made an important improvement towards equality between men and women. But the mentalities and the attitudes are still influenced by a traditional perception which depends on their social and cultural heritage. These perceptions are direct and indirect, transmitted to younger generations in particular through the textbooks. In this paper we studying what gender representations are valued in school textbooks. Our data were collected from a series of mathematic school handbooks used in primary school in Tunisia on 2003-2004.

INDEX

Mots-clés : genre, manuel scolaire, mathématiques, représentations sexuées, Tunisie

Keywords : gender representations, mathematic, textbook, Tunisia

\section{AUTEURS}

IBTIHEL BOUCHOUCHA

INED/CERPOS, Paris, France

THÉRÈSE LOCOH

INED, Paris, France 